\title{
Lung function, COPD and cognitive function: a multivariable and two sample Mendelian randomization study
}

\author{
Daniel H. Higbee ${ }^{1,2}$, Raquel Granell ${ }^{1}$, Gibran Hemani ${ }^{1}$, George Davey Smith ${ }^{1}$ and James W. Dodd ${ }^{1,2^{*}}$ (1)
}

\begin{abstract}
Background: Observational studies show an association between reduced lung function and impaired cognition. Cognitive dysfunction influences important health outcomes and is a precursor to dementia, but treatments options are currently very limited. Attention has therefore focused on identifying modifiable risk factors to prevent cognitive decline and preserve cognition. Our objective was to determine if lung function or risk of COPD causes reduced cognitive function using Mendelian randomization (MR).
\end{abstract}

Methods: Single nucleotide polymorphisms from genome wide association studies of lung function and COPD were used as exposures. We examined their effect on general cognitive function in a sample of 132,452 individuals. We then performed multivariable MR (MVMR), examining the effect of lung function before and after conditioning for covariates.

Results: We found only weak evidence that reduced lung function (Beta - 0.002 (SE 0.02), p-value 0.86) or increased liability to COPD (- 0.008 (0.008), $p$-value 0.35$)$ causes lower cognitive function. MVMR found both reduced FEV $V_{1}$ and FVC do cause lower cognitive function, but that after conditioning for height $(-0.03(0.03), p$-value 0.29 and -0.01 (0.03) $p$-value 0.62 , for FEV1 and FVC respectively) and educational attainment ( $-0.03(0.03) p$-value 0.33 and -0.01 (0.02), $p$-value 0.35 ) the evidence became weak.

Conclusion: We did not find evidence that reduced lung function or COPD causes reduced cognitive function. Previous observational studies are probably affected by residual confounding. Research efforts should focus on shared risk factors for reduced lung function and cognition, rather than lung function alone as a modifiable risk factor.

\section{Introduction}

Cognitive function impacts important physical and mental health outcomes including mortality and educational attainment [1]. It exists on a continuum from normal cognitive function, to the potentially reversible state of mild cognitive impairment (MCI), which can lead to irreversible dementia $[2,3]$. There are very limited therapeutic options which effectively increase cognitive

\footnotetext{
${ }^{*}$ Correspondence: James.dodd@bristol.ac.uk

${ }^{1}$ MRC Integrative Epidemiology Unit (IEU), University of Bristol, Oakfield Grove, Bristol BS8 2BN, UK

Full list of author information is available at the end of the article
}

function or treat $\mathrm{MCI}$, so finding modifiable risk factors is important.

Lung function measures such as Forced Expiratory Volume in one second $\left(\mathrm{FEV}_{1}\right)$ and Forced Expiratory Volume (FVC) have been linked with co-morbidities as early as the third decade [4]. There is also a growing body of evidence across cross sectional and longitudinal studies pointing to a an association between reduced lung function, Chronic Obstructive Pulmonary Disease (COPD) and cognitive function [5-10].

Co-morbid lung disease and cognitive impairment is associated with worse health outcomes, quality of life and increased healthcare utilisation [11]. The association 
between lung function and cognition may be due to shared risk factors seen more commonly in those with lung disease e.g. smoking $[12,13]$. However associations independent of these factors mediated through plausible causal pathological pathways such as hypoxia, hypercapnia or chronic lung disease associated inflammation may also cause extra pulmonary end organ damage [5, 14].

Neuroimaging provides further evidence of a relationship between lung function and cognition. After adjustment for smoking, reduced lung function remains associated with white matter brain lesions [15], and a 'dose response' like relationship is seen between severity of lung function deficit and risk of cognitive impairment [6].

Mendelian randomization (MR) is a method which can overcome problems of unmeasured confounding and reverse causation typical of conventional observational epidemiology [16]. MR allows causal inference through the use of genetic variants as proxies for modifiable risk factors and health outcomes [17]. MR has multiple advantages, it is not influenced by behavioural or environmental factors and minimizes reverse causation [17]. Additionally, the effects are equivalent to lifetime differences, reducing issues relating to transient fluctuations in exposures [18]. Multivariable MR (MVMR) has further advantages, it includes multiple exposures in the model (e.g. lung function and educational attainment) allowing estimation of the direct causal effect of each exposure on the outcome. MVMR is a robust method when using two exposures that could act as a confounders [19]. MR has been used in this way to show that low FVC (but not $\mathrm{FEV}_{1}$ ) causes coronary artery disease [20].

Our objective was to use MR and MVMR to determine if lung function or liability to COPD causes lower general cognitive function. If the relationship is shown to be causal, then interventions to treat lung function could also be used to reduce subsequent cognitive decline or dementia.

\section{Methods}

\section{Exposure populations}

The largest available lung function Genome Wide Association Study (GWAS) by Shrine et al. was utilised, which reported 279 Single Nucleotide Polymorphisms (SNPs) to $p$-value $<5 \times 10^{-9}[21]$. Lung function measurements used were $\mathrm{FEV}_{1}, \mathrm{FVC}, \mathrm{FEV}_{1} / \mathrm{FVC}$ and Peak Expiratory Flow (PEF). The GWAS was adjusted for age, age ${ }^{2}$, height and smoking status [21].

For liability to COPD, we used 82 SNPs identified from a GWAS of 35,735 COPD and 222,076 control subjects [22]. Cases were defined as those with pre-bronchodilator spirometry of $\mathrm{FEV}_{1} / \mathrm{FVC}<0.7$ and $\mathrm{FEV}_{1}$ percent predicted $<80 \%$. The GWAS was adjusted for age, age ${ }^{2}$, sex, height, principal components, and smoking. These two GWAS were used for 2-sample MR studies (2 sample MR is when the exposure and outcome GWAS are from two different sample populations).

The lung function GWAS methodology adjust for covariates of lung function and cognition e.g. height and smoking [12, 23]. This adjustment can lead to collider bias as SNPs can be related to lower levels of the covariates e.g. height, or to other adverse risk factors [18]. This can result in misleading SNP effect estimates and subsequent bias in MR studies [24]. To avoid these types of bias we performed MVMR using exposure SNPs discovered in GWAS that had not been adjusted for covariates. To find suitable exposure SNPs we performed a GWAS (adjusting for sex) based on 353,315 UK BioBank (UKBB) participants for "best measure" FEV 1 and FVC. We did not adjust for age as this is not genetically determined and allele effects are independent of age. The SNPs discovered in the unadjusted GWAS were used in a two-sample MVMR model conditioning on SNPs for covariates: standing height, body mass index (BMI), current smoking. Educational attainment is a significant determinant of cognitive function, but it was not adjusted for in the outcome GWAS [25]. Therefore, we used SNPs for a GWAS of the UK Biobank variable "at what age did you complete your continuous full time education", henceforth referred to as educational attainment. Participants were asked this via a touchscreen during assessment. By conditioning for educational attainment, we determined the direct effect of lung function on cognitive function. SNPs for these covariates were found in pre-existing GWAS performed in the UKBB [26]. See Additional files 1 and 2.

\section{Outcome population}

Data from a meta-analysis of the Cohort of Heart and Aging Research in Genomic Epidemiology (CHARGE) and Cognitive Genomics Consortium (COGENT) was used [1]. UKBiobank participants were excluded from our analysis to ensure no overlap of exposure and outcome populations. This resulted in an outcome population of 132,452. The CHARGE and COGENT cohorts constructed a general cognitive function phenotype a number of cognitive tasks. The general cognitive function phenotype required the cohorts to have tested at least three different cognitive domains. As different tasks were used across the different sample populations, a consistent method of extracting general cognitive function was used. Principal component analysis was applied to the cognitive test scores to derive a measure of general cognitive function. The CHARGE and COGENT cohorts avoided taking more than one cognitive score from any individual cognitive test. GWAS Meta-analysis adjusting 
for age, sex and population stratification was performed using the derived measure of general cognitive function. Therefore, the outcome used in our analysis derived from the CHARGE and COGENT cohorts represents general cognitive function and is not domain specific. For full details of the tasks and samples see the supplementary information of the reference [1].

Both exposure and outcome populations were in those of European ancestry. For a summary of the UK Biobank population please see Additional file 1: Table e4.

\section{Statistical analysis}

Statistical analysis was done using R Studio version 3.6.1 with MRCIEU/TwoSampleMR and MRInstruments packages $[19,27]$.

An assumption of Mendelian randomization is that the exposure SNPs are strongly associated with the exposure trait, in this case lung function and COPD. If the SNPs are only weakly associated with their respective exposure, this causes weak instrument bias and may invalidate the analysis. Therefore, F-statistics were calculated to assess exposure instruments strength (F statistic $=$ beta $^{2} /$ standard error ${ }^{2}$ ). If the F-statistic is $>10$ it means weak instrument bias in unlikely, and the higher the F-statistic the lower the chance of weak instrument bias [28]. For all exposures SNPs LD-clumping was performed using European reference population and the ieugwasr:ld clump tool. This removes SNPs that are in close proximity $(\mathrm{kb}=10,000)$, or highly correlated $\left(\mathrm{r}^{2} 0.001\right)$, to ensure that all SNPs used have independent effects. Steiger filtering was performed, to remove variants that caused more variance of the outcome than the exposure [27]. Duplicate SNPs were removed. Palindromic SNPs (i.e. $\mathrm{A} / \mathrm{T}$ and $\mathrm{C} / \mathrm{G}$ SNPs) with intermediate allele frequencies were excluded from analysis to ensure that the correct allele was being used. All SNPs were harmonised to ensure the exposure and outcome alleles were the same. See Additional files 1 and 2.

Inverse Variance Weighting (IVW) was used for main effect estimate for all analyses. IVW is a weighted regression of SNP-outcome on SNP-exposure associations combined.

MR assumptions and details of sensitivity tests used are detailed in the Additional files 1 and 2.

To account for the possibility of horizontal pleiotropy (where SNPs influence both exposure and outcome through independent pathways), in the $2 S-M R$ analysis we performed MR Egger. Similar to IVW, MR-Egger is a weighted regression of SNP-outcome on SNP-exposure associations, but the intercept is not fixed to zero. We used weighted median and mode MR to minimise the effect of unbalanced instruments on an overall estimate of the mean. Heterogeneity (the variability in causal estimates obtained for each SNP) is an indication of potential violation of assumptions. This was calculated and assessed with a $\mathrm{Q}$ statistic, presented as a $Q_{-} P$-value. MR Radial was performed to identify and exclude outliers and re-estimate IVW. MR radial removes SNPs that contribute more than $5 \%$ to the heterogeneity of the model [29].

IRB approval was not required as we did not recruit individuals but used publicly accessible data. UK Biobank is monitored by the UK Biobank Ethics and Governance Council.

\section{Results}

The analysis using the adjusted lung function GWAS in a 2-sample lung function analysis is reported in Table 1 [21]. Effects are per standard deviation (SD) decrease in lung function measure. F statistic for all SNPs combined for each lung function trait were $=111, \mathrm{FEV}_{1}=69$, $\mathrm{FVC}=70, \mathrm{FEV}_{1} / \mathrm{FVC}=148$, making weak instrument bias unlikely. The predictive causal effects show reduced $\mathrm{FEV}_{1}$ and $\mathrm{FVC}$ reduced cognition across all tests, but the evidence was weak (Table 1). All lung function measures combined did not show consistent direction of effect across the tests used. There was strong evidence of heterogeneity of effect based on the Q_P-value, especially when assessing all measures combined. However, there were no visual outliers (Additional file 1: Figures E1-E4) and minimal change in effect estimates after using MRRadial to exclude outliers and recalculation of IVW. See Additional files 1 and 2.

F statistic for COPD SNPs combined is 52, making weak instrument bias unlikely. There is a consistent direction of effect that increased liability to COPD causes lower cognition, however the strength of evidence is weak (Table 2). There was evidence of heterogeneity, however no outliers were identified visually (Additional file 1: Figures E5-E8) and exclusion of outliers in MRRadial only minimally changed effect estimates.

\section{MVMR analysis}

Using a threshold of $p<5 \times 10^{-8}$, quality control and clumping the unadjusted GWAS of lung function in UKBB produced 360 SNPs for $\mathrm{FEV}_{1}$ and 464 SNPs for FVC explaining $3.6 \%$ and $4.8 \%$ of variance, respectively. Height (Beta 0.05 (SE 0.01), $p$-value $<0.001)$, BMI $(-0.1$ (0.01), $p$-value $<0.001)$ and educational attainment $(0.3$ (0.07), $p$-value $<0.001)$ showed strong evidence for an effect on cognitive function. The SNPs discovered in the GWAS of current smoking only had very weak evidence of effect on cognitive function $(-0.04(0.2), p$-value 0.84$)$, so they were not included in the analysis. The F-statistic for combined SNPs for each lung function trait are $\mathrm{FEV}_{1}=38, \mathrm{FVC}=40$, standing height $=50, \mathrm{BMI}=39$ and 
Table 1 2-Sample MR, decreasing lung function effect on cognitive function

\begin{tabular}{|c|c|c|c|c|c|}
\hline \multirow[t]{2}{*}{ Lung function measure } & \multirow[t]{2}{*}{ No. of SNPs } & \multirow[t]{2}{*}{ Test used } & \multicolumn{3}{|c|}{ Cognitive function } \\
\hline & & & Beta (SE) & $p$-value & Q_P-value \\
\hline \multirow[t]{4}{*}{$\mathrm{FEV}_{1}, \mathrm{FVC}, \mathrm{FEV}_{1} / \mathrm{FVC}, \mathrm{PEF}$} & 173 & IVW & $-0.002(0.02)$ & 0.86 & $1.44 \times 10^{11}$ \\
\hline & & Weighted median & $0.02(0.02)$ & 0.44 & \\
\hline & & Weighted Mode & $-0.004(0.03)$ & 0.91 & \\
\hline & & MR Egger & $-0.005(0.03)$ & 0.90 & \\
\hline \multirow[t]{4}{*}{$\mathrm{FEV}_{1}$} & 59 & IVW & $-0.05(0.04)$ & 0.44 & $1.8 \times 10^{-8}$ \\
\hline & & Weighted median & $-0.02(0.04)$ & 0.46 & \\
\hline & & Weighted mode & $-0.05(0.08)$ & 0.51 & \\
\hline & & MR Egger & $-0.09(0.1)$ & 0.43 & \\
\hline \multirow[t]{4}{*}{ FVC } & 68 & IVW & $-0.004(0.03)$ & 0.87 & 0.01 \\
\hline & & Weighted median & $-0.02(0.04)$ & 0.51 & \\
\hline & & Weighted mode & $-0.03(0.07)$ & 0.65 & \\
\hline & & MR Egger & $-0.16(0.10)$ & 0.13 & \\
\hline \multirow[t]{4}{*}{$\mathrm{FEV}_{1} / \mathrm{FVC}$} & 93 & IVW & $0.01(0.02)$ & 0.52 & 0.01 \\
\hline & & Weighted median & $0.02(0.02)$ & 0.52 & \\
\hline & & Weighted mode & $0.007(0.05)$ & 0.87 & \\
\hline & & MR Egger & $0.02(0.04)$ & 0.67 & \\
\hline
\end{tabular}

Negative beta indicates decreasing cognitive function

$\mathrm{SE}$, standard error; $\mathrm{Q}_{-} P$-value, a measure of heterogeneity ( $p$-value $<0.05$ provides strong evidence of heterogeneity)

Table 2 2-Sample MR, COPD effect on cognitive function

\begin{tabular}{|c|c|c|c|c|c|}
\hline \multirow[t]{2}{*}{ Lung function measure } & \multirow[t]{2}{*}{ No. of SNPs } & \multirow[t]{2}{*}{ Test used } & \multicolumn{3}{|c|}{ Cognitive function } \\
\hline & & & Beta (SE) & $p$-value & Q_P-value \\
\hline \multirow[t]{4}{*}{ COPD } & 67 & IVW & $-0.008(0.008)$ & 0.35 & 0.005 \\
\hline & & Weighted median & $-0.01(0.01)$ & 0.16 & \\
\hline & & Weighted mode & $-0.03(0.02)$ & 0.28 & \\
\hline & & MR Egger & $-0.01(0.02)$ & 0.66 & \\
\hline
\end{tabular}

Negative beta indicates decreasing cognitive function

age completed full time education $=1350$, making weak instrument bias unlikely.

Results were calculated per SD decrease in lung function measure. There was strong evidence that reduced $\mathrm{FEV}_{1}(-0.06$ (0.03), $p$-value $<0.001)$ and FVC $(-0.06$ (0.01), $p$-value $<0.001)$ cause lower general cognition, however with MVMR after conditioning with educational attainment the evidence became weak. Evidence also became weak after conditioning for height. This is probably due to the pleiotropy in the MR analysis as the unadjusted GWAS would have discovered SNPs that affected LF via height or educational attainment (Table 3).

\section{Discussion}

Our analyses show weak evidence that lung function or liability to COPD causes lower general cognitive function. Most causal estimates show the same direction of
Table 3 MVMR analysis results

\begin{tabular}{|c|c|c|c|c|}
\hline \multirow{2}{*}{$\begin{array}{l}\text { Lung } \\
\text { function } \\
\text { measure }\end{array}$} & \multirow[t]{2}{*}{ Condition } & \multirow{2}{*}{$\begin{array}{l}\text { No SNPs } \\
\text { (trait/ } \\
\text { condition) }\end{array}$} & \multicolumn{2}{|c|}{ Cognitive function } \\
\hline & & & Beta (SE) & $p$-value \\
\hline $\mathrm{FEV}_{1}$ & None & $298 / 0$ & $-0.06(0.03)$ & $<0.001$ \\
\hline $\mathrm{FEV}_{1}$ & Height & $212 / 299$ & $-0.03(0.03)$ & 0.29 \\
\hline $\mathrm{FEV}_{1}$ & $\mathrm{BMl}$ & $180 / 629$ & $-0.07(0.03)$ & 0.03 \\
\hline $\mathrm{FEV}_{1}$ & $\begin{array}{l}\text { Educational } \\
\text { attainment }\end{array}$ & $274 / 32$ & $-0.03(0.03)$ & 0.33 \\
\hline FVC & None & $381 / 0$ & $-0.06(0.01)$ & $<0.001$ \\
\hline FVC & Height & $278 / 314$ & $-0.01(0.03)$ & 0.62 \\
\hline FVC & $\mathrm{BMI}$ & $224 / 595$ & $-0.05(0.02)$ & 0.004 \\
\hline FVC & $\begin{array}{l}\text { Educational } \\
\text { attainment }\end{array}$ & $335 / 31$ & $-0.01(0.02)$ & 0.35 \\
\hline
\end{tabular}

Effect of decreasing Lung function trait on cognitive function with and without conditioning for covariates

Negative beta indicates decreasing cognitive function

SE, standard error 
effect as observational studies, with worse lung function, liability to COPD causing lower general cognitive function, however the evidence is weak. The observed association is likely secondary to residual confounding and collider bias. This would indicate that observed associations may be present but not causal [5-10].

Two-sample MR eliminates many confounders in observational epidemiology $[17,30]$. We used a large number of robust lung function SNPs, and SNPs that influence liability to COPD, which have been well validated in large samples [21, 22]. MVMR was utilised to decrease the risk of bias and allowed us to condition for the effect of educational attainment. The effects of instrumental variables were assessed in a huge outcome population of similar age and the same ancestral population as our discovery GWAS.

Our analysis suggest that shared risk factors are likely explanations for the observed association between lung function and cognition, for example cigarette smoking. The short term effects of smoking on cognitive function are complex with acute nicotine consumption improving smokers' cognition, and nicotine abstinence decreasing cognition [12]. Longitudinal research has shown that lower childhood IQ is associated with increased risk of smoking, and smokers have significantly worse cognition scores in old age than ex- or never-smokers [13]. Therefore, public health measures to reduce rates of smoking could improve both lung function and cognitive function.

Both cognition and lung function follow life course trajectories, influenced by a combination of genetic and life course factors [4]. Genetic determinants of lung development and disease are increasingly recognised [31]. In addition to shared environmental risk for lung function and cognitive function, shared genetic risks may be found. Genes involved in growth factors and Vitamin A regulation have been found to affect lung function. Both growth patterns and Vitamin A levels may have a role in cognitive function [23, 32]. It may be that genetic pleiotropy can determine both lung function and cognitive trajectories.

\section{Limitations}

This study examines the effect of lung function and COPD on general cognitive function in general adult population. COPD has been shown to be associated with general cognitive function, but often more specific patterns of cognitive impairment including attention, memory, learning and motor function domains [5]. Therefore it may be that COPD has does have causal effect on these domains, not detected in our general cognitive function analysis.

This is comprehensive and robust analysis of causal association between lung function and risk of COPD, which uses two exposures and MVMR methodology. It is worth noting the SNPs in one of our two exposure analysis refers to 'liability' rather than confirmed diagnosis of COPD as no SNP guarantees future COPD, but MR is still a valid test of the causal null hypothesis for a binary exposure [33]. The mean age in our outcome sample was 56, lower than the average age of COPD diagnosis. The effects of SNPs for binary traits may be underestimated in the outcome sample, as participants have not yet developed COPD or its co-morbidities. However, the exposure SNPs were discovered and validated in large populations that had similar mean ages [21, 22]. Therefore the SNPs' estimated effects on COPD liability should be accurate in the outcome population. If the mean age of the outcome population was older, it could introduce a survivor bias if a proportion of those with the SNPs had died from COPD and could not be recruited for the outcome GWAS [34].

One of the proposed mechanisms whereby lung function and COPD may cause reduced cognition is via cerebrovascular pathology [35]. Our outcome population excluded those with history of clinical stroke, this is unlikely to have excluded the proposed COPD specific brain changes, but would have excluded those with large vessel vascular damage causing changes in cognitive function.

Cognitive function can be correlated to factors that distort effect estimates. These include demography, (when a populations genetic variance is related to geographical location), assortative mating (partners are chosen due to phenotypes e.g. higher cognitive function, rather than randomly) and dynastic effects (phenotypic expression of parents genotype affects offspring phenotype e.g. parents with higher education giving educational books to their children) [36]. This can be corrected for by using within-family GWAS, a possible area for future studies [37]. However, MR studies have tended to over-estimate the effect of anthropomorphic traits which then attenuate when using within-family studies, and in this study we only found weak evidence of an anthropomorphic trait having an effect.

The populations used by the GWAS for exposure SNP's only used European ancestry participants. These results may therefore not be generalizable to non-European ancestral populations. We did not have a replication cohort for the SNPs used in the MVMR analysis. The effects of the SNPs could have been over estimated due to "Winner's Curse" phenomenon [38]. This occurs in GWAS when effect estimates are biased towards the SNPs with the strongest association. However, this phenomenon would bias results away from the null. 


\section{Future research}

Our outcome GWAS was performed with global cognitive function in the general population as a continuous outcome. Much research has focused on whether lung function and lung disease causes mild cognitive impairment (MCI) [39]. Normal cognitive function and MCI exist on a spectrum, but our study is unable to fully assess whether reduced lung function or lung disease causes MCI. If a GWAS of MCI becomes available MR could be used in future studies.

Our results indicate that lung function alone does not causes lower cognition in general population. However, lower lung function and lung disease have been shown to be associated with reduced cognitive function, but this is most likely due to shared risk factors. Research should focus on reducing exposure to these shared risk factors and optimising the management of co-morbidities in those with chronic lung disease.

\section{Conclusion}

Our study shows that lung function and COPD do not cause reduced cognition in the general population. Previous observational studies suggesting a causal link were probably affected by residual confounding. The observed associations between reduced lung function, COPD and cognitive function remain important. Research should now focus on the management of cognitive impairment in these groups, rather than targeting lung function alone in order to improve cognition in the general population.

\section{Supplementary Information}

The online version contains supplementary material available at https://doi. org/10.1186/s12890-021-01611-6.

Additional file 1. Supplementary information.

Additional file 2. SNP lists.

\section{Acknowledgements}

This research was conducted using UKBiobank resource (project number 55521). We would like to thank all UKBiobank participants and all staff involved in UKBiobank. We would like to thank Gail Davies and lan Deary for providing the outcome GWAS with UKBiobank population excluded.

\section{Authors' contributions}

All authors contributed to analysis, interpretation and final manuscript preparation, JWD, DHH contributed to study concept, rationale, and initial manuscript drafts. All authors read and approved the final manuscript.

\section{Funding}

This work was supported by the Medical Research Council and the University of Bristol (MC_UU_00011). MRC CARP Fellowship. (MR/T005114/1).

\section{Availability of data and materials}

All exposure SNPs used are available in the Additional file 2. They are also available online at the MRC IEU repository, https://gwas.mrcieu.ac.uk. ieu-b-106, ieu-b-105, ieu-b-104.
Ethics approval and consent to participate

Ethical approval and consent was not specifically for this study as we used summary data that is publicly available.

\section{Competing interests}

JWD declares personal fees from Chiesi, Boerhinger Ingelheim, AstraZeneca and GSK. None have relevance to this paper. The other authors have no competing interests to declare.

\section{Author details}

${ }^{1} \mathrm{MRC}$ Integrative Epidemiology Unit (IEU), University of Bristol, Oakfield Grove, Bristol BS8 2BN, UK. ${ }^{2}$ Academic Respiratory Unit, Southmead Hospital, University of Bristol, Bristol BS10 5NB, UK.

Received: 17 May 2021 Accepted: 8 July 2021

Published online: 22 July 2021

\section{References}

1. Davies G, Lam M, Harris SE, Trampush JW, Luciano M, Hill WD, Hagenaars SP, Ritchie SJ, Marioni RE, Fawns-Ritchie C, et al. Study of 300,486 individuals identifies 148 independent genetic loci influencing general cognitive function. Nat Commun. 2018:9(1):2098-2098.

2. Sanford AM. Mild cognitive impairment. Clin Geriatr Med. 2017:33(3):325-37.

3. Jongsiriyanyong S, Limpawattana P. Mild cognitive impairment in clinical practice: a review article. Am J Alzheimers Dis Other Demen. 2018;33(8):500-7.

4. Agusti A, Noell G, Brugada J, Faner R. Lung function in early adulthood and health in later life: a transgenerational cohort analysis. Lancet Respir Med. 2017;5(12):935-45.

5. Dodd JW. Lung disease as a determinant of cognitive decline and dementia. Alzheimer's Res Therapy. 2015;7(1):32.

6. Brunner EJ, Welch CA, Shipley MJ, Ahmadi-Abhari S, Singh-Manoux A, Kivimäki M. Midlife risk factors for impaired physical and cognitive functioning at older ages: a cohort study. J Gerontol A Biol Sci Med Sci. 2017;72(2):237-42.

7. Suglia SF, Wright RO, Schwartz J, Wright RJ. Association between lung function and cognition among children in a prospective birth cohort study. Psychosom Med. 2008;70(3):356-62.

8. Wen XH, Li Y, Han D, Sun L, Ren PX, Ren D. The relationship between cognitive function and arterial partial pressure $\mathrm{O} 2$ in patients with COPD: a meta-analysis. Medicine. 2018;97(4):e9599.

9. Liesker JJ, Postma DS, Beukema RJ, ten Hacken NH, van der Molen T, Riemersma RA, van Zomeren EH, Kerstjens HA. Cognitive performance in patients with COPD. Respir Med. 2004;98(4):351-6.

10. Bajaj MK, Burrage DR, Tappouni A, Dodd JW, Jones PW, Baker EH. COPD patients hospitalized with exacerbations have greater cognitive impairment than patients hospitalized with decompensated heart failure. Clin Interv Aging. 2019;14:1-8.

11. Chang SS, Chen S, McAvay GJ, Tinetti ME. Effect of coexisting chronic obstructive pulmonary disease and cognitive impairment on health outcomes in older adults. J Am Geriatr Soc. 2012;60(10):1839-46.

12. Campos MW, Serebrisky D, Castaldelli-Maia JM. Smoking and Cognition. Curr Drug Abuse Rev. 2016;9(2):76-9.

13. Corley J, Gow AJ, Starr JM, Deary IJ. Smoking, childhood IQ, and cognitive function in old age. J Psychosom Res. 2012;73(2):132-8.

14. Austin V, Crack PJ, Bozinovski S, Miller AA, Vlahos R. COPD and stroke: are systemic inflammation and oxidative stress the missing links? Clin Sci (Lond). 2016:130(13):1039-50.

15. Liao D, Higgins M, Bryan NR, Eigenbrodt ML, Chambless LE, Lamar V, Burke GL, Heiss G. Lower pulmonary function and cerebral subclinical abnormalities detected by MRI: the Atherosclerosis Risk in Communities study. Chest. 1999;116(1):150-6.

16. Smith GD, Ebrahim S. "Mendelian randomization": can genetic epidemiology contribute to understanding environmental determinants of disease? Int J Epidemiol. 2003;32(1):1-22.

17. Davey Smith G, Hemani G. Mendelian randomization: genetic anchors for causal inference in epidemiological studies. Hum Mol Genet. 2014;23(R1):R89-98. 
18. Davey Smith G, Paternoster L, Relton C. When will mendelian randomization become relevant for clinical practice and public health? Mendelian randomization and clinical practice and public HealthEditorial. JAMA. 2017;317(6):589-91.

19. Sanderson E, Davey Smith G, Windmeijer F, Bowden J. An examination of multivariable Mendelian randomization in the single-sample and twosample summary data settings. Int J Epidemiol. 2018;48(3):713-27.

20. Higbee DH, Granell R, Sanderson E, Davey Smith G, Dodd JW. Lung function \& cardiovascular disease. A two sample Mendelian randomisation study. Eur Respir J. 2021;2003196.

21. Shrine N, Guyatt AL, Erzurumluoglu AM, Jackson VE, Hobbs BD, Melbourne CA, Batini C, Fawcett KA, Song K, Sakornsakolpat P, et al. New genetic signals for lung function highlight pathways and chronic obstructive pulmonary disease associations across multiple ancestries. Nat Genet. 2019;51(3):481-93.

22. Sakornsakolpat P, Prokopenko D, Lamontagne M, Reeve NF, Guyatt AL Jackson VE, Shrine N, Qiao D, Bartz TM, Kim DK, et al. Genetic landscape of chronic obstructive pulmonary disease identifies heterogeneous celltype and phenotype associations. Nat Genet. 2019;51(3):494-505.

23. Cohen-Manheim I, Doniger GM, Sinnreich R, Simon ES, Murad H, PinchasMizrachi R, Kark JD. Body mass index, height and socioeconomic position in adolescence, their trajectories into adulthood, and cognitive function in midlife. J Alzheimers Dis. 2017;55(3):1207-21.

24. Aschard H, Vilhjálmsson Bjarni J, Joshi Amit D, Price Alkes L, Kraft P. Adjusting for heritable covariates can bias effect estimates in genome-wide association studies. Am J Hum Genet. 2015;96(2):329-39.

25. Leggett A, Clarke P, Zivin K, McCammon RJ, Elliott MR, Langa KM. Recent improvements in cognitive functioning among older U.S. adults: how much does increasing educational attainment explain? J Gerontol B Psychol Sci Soc Sci. 2019;74(3):536-45.

26. https://gwas.mrcieu.ac.uk/. 2020.

27. Hemani G, Tilling K, Davey Smith G. Orienting the causal relationship between imprecisely measured traits using GWAS summary data. PLoS Genet. 2017;13(11):e1007081.

28. Burgess S, Thompson SG, Collaboration CCG. Avoiding bias from weak instruments in Mendelian randomization studies. Int J Epidemiol. 2011;40(3):755-64.

29. Bowden J, Spiller W, Del Greco MF, Sheehan N, Thompson J, Minelli C, Davey Smith G. Improving the visualization, interpretation and analysis of two-sample summary data Mendelian randomization via the Radial plot and Radial regression. Int J Epidemiol. 2018;47(4):1264-78.

30. Smith GD, Ebrahim S. What can mendelian randomisation tell us about modifiable behavioural and environmental exposures? BMJ. 2005;330(7499):1076-9.

31. Portas L, Pereira M, Shaheen SO, Wyss AB, London SJ, Burney PGJ, Hind M, Dean $\mathrm{CH}$, Minelli C. Lung development genes and adult lung function. Am J Respir Crit Care Med. 2020;202(6):853-65.

32. Wołoszynowska-Fraser MU, Kouchmeshky A, McCaffery P. Vitamin A and retinoic acid in cognition and cognitive disease. Annu Rev Nutr. 2020:40:247-72.

33. Burgess S, Labrecque JA. Mendelian randomization with a binary exposure variable: interpretation and presentation of causal estimates. Eur J Epidemiol. 2018;33(10):947-52.

34. Vansteelandt S, Dukes O, Martinussen T. Survivor bias in Mendelian randomization analysis. Biostatistics. 2017;19(4):426-43.

35. Spilling CA, Bajaj MPK, Burrage DR, Ruickbie S, Thai NJ, Baker EH, Jones PW, Barrick TR, Dodd JW. Contributions of cardiovascular risk and smoking to chronic obstructive pulmonary disease (COPD)-related changes in brain structure and function. Int J Chron Obstruct Pulmon Dis. 2019;14:1855-66

36. Trejo S, Domingue BW. Genetic nature or genetic nurture? Introducing social genetic parameters to quantify bias in polygenic score analyses. Biodemography Soc Biol. 2018;64(3-4):187-215.

37. Brumpton B, Sanderson E, Heilbron K, Hartwig FP, Harrison S, Vie G, Cho $Y$, Howe LD, Hughes A, Boomsma Dl, et al. Avoiding dynastic, assortative mating, and population stratification biases in Mendelian randomization through within-family analyses. Nat Commun. 2020;11(1):3519.

38. Kraft P. Curses — winner's and otherwise —in genetic epidemiology. Epidemiology. 2008;19(5):649-51.

39. Higbee DH, Dodd JW. Cognitive impairment in COPD: an often overlooked co-morbidity. Expert Rev Respir Med. 2021;15:9-11.

\section{Publisher's Note}

Springer Nature remains neutral with regard to jurisdictional claims in published maps and institutional affiliations.
Ready to submit your research? Choose BMC and benefit from:

- fast, convenient online submission

- thorough peer review by experienced researchers in your field

- rapid publication on acceptance

- support for research data, including large and complex data types

- gold Open Access which fosters wider collaboration and increased citations

- maximum visibility for your research: over $100 \mathrm{M}$ website views per year

At BMC, research is always in progress.

Learn more biomedcentral.com/submissions 